\title{
Mathematical Methods for the Analysis of Color Scanning Filters
}

\author{
Poorvi L. Vora and H. Joel Trussell, Fellow, IEEE
}

\begin{abstract}
The problem of the sensitivity analysis of color scanning filters is addressed in this paper. The second differential of the mean square $\Delta E_{a b}$ error provides a means of calculating the sensitivity of the mean square $\Delta E_{a b}$ error to filter fabrication errors. Tolerances on the allowable change in the mean square $\Delta E_{a b}$ error are used to define bounds on the filter fabrication errors at all wavelengths and at single wavelengths.
\end{abstract}

\section{INTRODUCTION}

I N A COMPANION paper a method for the design of color scanning filters is presented [7]. This paper will consider the effects of errors that are inevitable when fabricating a desired filter. To begin, let us briefly review the mathematical basis and notation used in [7].

The color for a radiant spectrum is determined by

$$
\mathbf{t}=\mathbf{A}^{T} \mathbf{r}
$$

where $\mathbf{r}$ is an $N$-vector representing the sampled spectrum of a radiant source, $\mathbf{A}$ is an $N \times 3$ matrix representing the Commission Internationale de L'Eclairage (CIE) color matching functions, and $\mathbf{t}$ is a 3-vector representing the CIE tristimulus values. A reflectance spectrum can be measured by producing a radiant spectrum

$$
\mathbf{r}=\mathbf{L f}
$$

where $\mathbf{f}$ is a reflectance spectrum and $\mathbf{L}$ is an $N \times N$ diagonal matrix representing the spectrum under which the reflecting object is viewed. The color matching functions and the illuminant can be combined in a single matrix, $\mathbf{A}_{L}=\mathbf{L A}$, which defines the human visual subspace (HVSS) under the illuminant $\mathbf{L}$.

The problem of designing filters for measuring the color is formulated in [7] as finding a set of vectors that span the HVSS. The problem can be extended to finding a set of vectors that span any desired subspace. The measurements taken with the actual filters are given by

$$
\mathbf{c}=\mathbf{M}^{T} \mathbf{H f}
$$

Manuscript received January 12, 1994; revised January 6, 1996. The associate editor coordinating the reivew of this paper and approving it for publication was Dr. Fredrick Mintzer.

P. L. Vora is with Hewlett-Packard Laboratories, Palo Alto, CA 94304 USA.

H. J. Trussell is with the Department of Electrical and Computer Engineering, North Carolina State University, Raleigh, NC 27695-7911 USA (e-mail: hjt@ecehjt.ncsu.edu).

Publisher Item Identifier S 1057-7149(97)00994-9. where $\mathbf{M}$ represents the scanning filters, $\mathbf{H}$ is a diagonal matrix representing the spectral effects of the detectors, the optical path, and the spectrum used for illuminating the reflecting object, and $\mathbf{c}$ represents the resulting measurement. The motivation for using a parameterized description of the filters in the design problem was that the fabrication process requires transmission functions that are relatively smooth [7]. These designed filters will not span the required space, thus, it is important to measure the goodness of a nonideal filter set. The measure of the goodness [5] of the effective set $\mathbf{M}_{H}=\mathbf{H M}$ with respect to the desired space defined by $\mathrm{V}$ [7] is

$$
\begin{aligned}
& \nu\left(\mathbf{V}, \mathbf{M}_{H}\right)= \\
& \frac{\operatorname{Trace}\left[\mathbf{V}\left(\mathbf{V}^{T} \mathbf{V}\right)^{-1} \mathbf{V}^{T} \mathbf{M}_{H}\left(\mathbf{M}_{H}^{T} \mathbf{M}_{H}\right)^{-1} \mathbf{M}_{H}^{T}\right]}{\alpha}
\end{aligned}
$$

where $\alpha$ is the rank of the matrix $\mathbf{V}$.

The design method presented in the companion paper uses the measure of goodness $\nu$ of [5] as an optimization criterion and incorporates the constraint of smoothness of the filters into the design procedure. It is impossible to fabricate the designed "optimal" filters exactly. This manufacturing perturbation in filter transmissivities leads to a general degradation of filter performance indicated by larger average tristimulus errors, average $\Delta E_{a b}$ errors and smaller values of the measure $\nu$. The errors in the CIELAB space are important because they are closely related to human perceptual errors. The average square $\Delta E_{a b}$ error over a data set is defined as

$$
\begin{aligned}
E_{s q} & =\frac{\sum_{\mathbf{f}} \Delta E_{a b}^{2}(\mathbf{f})}{n} \\
= & \frac{\sum_{\mathbf{f}}\left[\left(L^{*}-L_{f}^{*}\right)^{2}+\left(a^{*}-a_{f}^{*}\right)^{2}+\left(b^{*}-b_{f}^{*}\right)^{2}\right]}{n}
\end{aligned}
$$

where $\sum_{\mathbf{f}}$ represents the sum over the data set, $\left[L^{*}, a^{*}, b^{*}\right]^{T}$ the transformed (estimated) tristimulus vector in CIELAB space [9] for data point $\mathbf{f}$ and a given viewing illuminant, and $\left[L_{f}^{*}, a_{f}^{*}, b_{f}^{*}\right]^{T}$ the transformed (actual) tristimulus vector in CIELAB space for the same point under the same viewing illuminant.

This paper derives quantitative estimates of the changes in $E_{s q}$ that result from perturbations of filter transmissivities. Such estimates are very useful for the manufacturers since they indicate the critical portions of the filters. Experimental data demonstrates the accuracy of the sensitivity estimates. 
Error modeling provides a basis for calculating worst-case bounds on filter fabrication errors, and bounds on maximum allowable filter fabrication errors as a function of wavelength are presented. As with the design method described in [7], the accuracy of the sensitivity analysis depends on the accuracy of scanner characterization.

Section II presents a method of obtaining a quantitative measure of the effect of small perturbations in the color scanning filters on $E_{s q}$ over a particular data set. It is demonstrated in Section III how the quantitative estimates can be used to define bounds on the allowable error in filter fabrication given a particular tolerance for maximum $E_{s q}$. Section IV presents simulation results to support the claims in Sections II and III. Conclusions are presented in Section V.

\section{ERROR ANALYSIS}

A small change in filter transmissivities will, in general, change the performance of the scanning filters. Because the performance sensitivity to changes in filter transmissivities can be measured in terms of the measure $\nu$ or the meansquare perceptual error $E_{s q}$, a general scalar function, $g$, will be used to describe the method for approximating change in performance. The Taylor series is used to approximate the change. The definitions and approximations associated with the Taylor series are presented to provide the mathematical background for the approximations and the sensitivity analysis that follows. The error function that is of interest in this work is a scalar function of a matrix that represents the color scanning filters.

Consider the Taylor formula for a scalar function of a single variable. For a scalar function $y$ of a single variable $x$, the first differential at $x_{0}$ due to a change $\delta x$ in the argument is the linear part of the increment in the function. It may be expressed [2, p. 81] as

$$
d y\left(x_{0} ; \delta x\right)=\left.\frac{\partial y}{\partial x}\right|_{x=x_{0} .} \delta x
$$

Notice that the first differential depends on both $x_{0}$ and $\delta x$, but that the term $\partial y /\left.\partial x\right|_{x=x_{0}}$ depends only on $x_{0}$. Hence, the first differential is linear in $\delta x$. Similarly, the second differential is a quadratic expression in $\delta x$. It can be written as

$$
d^{2} y\left(x_{0} ; \delta x\right)=\left.\frac{\partial^{2} y}{\partial^{2} x}\right|_{x=x_{0}}(\delta x)^{2}
$$

and is a function of both $x_{0}$ and $\delta x$. The second-order Taylor series approximation for the value of $y$ at a point $x+\delta x$ is

$$
y\left(x_{0}+\delta x\right) \approx y\left(x_{0}\right)+d y\left(x_{0} ; \delta x\right)+\frac{1}{2} d^{2} y\left(x_{0} ; \delta x\right) .
$$

The Taylor approximations of (6) can be extended to scalar functions of vector-valued arguments as follows.

The second-order Taylor formula may be used to approximate scalar function $g$ in the neighborhood of a vector-valued argument $x_{0}$ if $g$ is twice-differentiable at $x_{0}$ [2, p. 108]:

$$
g\left(x_{0}+\delta x\right)=g\left(x_{0}\right)+d g\left(x_{0} ; \delta x\right)+\frac{1}{2} d^{2} g\left(x_{0} ; \delta x\right)+r_{c}(\delta x)
$$

where $r_{c}(\delta x)$ is the residual error which has the property

$$
\lim _{\|\delta x\| \rightarrow 0} \frac{r_{c}(\delta x)}{\|\delta x\|^{2}}=0
$$

and $d g$ is the first differential of $g, d^{2} g$ the second differential. Both are functions of $x_{0}$ and $\delta x$, and defined by

$$
d g=\mathbf{j}^{T} \delta x
$$

where $\mathbf{j}_{i}=\partial g /\left.\partial x_{i}\right|_{x=x_{0}}$, and

$$
d^{2} g=(\delta x)^{T} \mathcal{H} \delta x
$$

for a symmetric matrix $\mathcal{H}$, the Hessian of $g$ [2].

The change in the scalar function $g$ when $x$ changes by a "small" amount $\delta x$ may be approximated [2] as

$$
\begin{aligned}
\delta g & =g\left(x_{0}+\delta x\right)-g\left(x_{0}\right) \\
& \approx d g\left(x_{0} ; \delta x\right)+\frac{1}{2} d^{2} g\left(x_{0} ; \delta x\right) .
\end{aligned}
$$

Given a scalar function of a matrix $\mathbf{X}$, the function may be written as a scalar function $g$ of the vector of stacked columns of $\mathbf{X}, \operatorname{vec}(\mathbf{X})$. In the particular application treated here, it is desired to approximate the change in $E_{s q}$ over a data set if the designed filters $\mathbf{M}$ are perturbed to give fabricated filters $\mathbf{M}+\Delta \mathbf{M}$. The scalar function $g$ will be replaced by $E_{s q}$ over a particular data set for the rest of this section.

For a set of $r$ scanning filters, each represented by $N$ samples of the transmittance functions, the function $g$ is a function of $r N$ variables. The first differential of $E_{s q}$ has been derived in [6]. When the designed filters are trimmed (optimized) with respect to $E_{s q}$ as described in [7] to produce filters that are close to optimal with respect to $E_{s q}$ over a data set with $\mathrm{n}$ spectra, the first differential $d E_{s q}$ will be close to zero. This means that the second term in (8) will be dominant in the expression for $\delta g$. The second differential of a scalar function of a matrix $\mathbf{X}[$ see (7)] is

$$
d^{2} g=(\operatorname{vec} d \mathbf{X})^{T} \mathcal{H} \operatorname{vec} d \mathbf{X}
$$

where $\mathcal{H}$ is the Hessian of the scalar function with respect to vecX. A mathematical expression for the second differential of $E_{s q}$ over a particular data set, and in particular an expression for the matrix $\mathcal{H}$, has been derived in [6] using methods described in [2]. Similar ideas may be used to obtain a mathematical expression for the second differential of the measure $\nu$. The expression may be found in [6].

Experimental results confirm that the second term, $\frac{1}{2}\left(d^{2} g\right)$, is a fairly good approximation to the observed change in $E_{s q}$, and that it contributes a much larger part to the change than does the first differential for the range of values of $\Delta \mathrm{M}$ under consideration. While all experimental results presented here deal with the second differential of $E_{s q}$ over a data set, the method described may be used to perform the sensitivity analysis of any scalar error measure with respect to changes in filter transmittances. In particular, it may be used to perform a sensitivity analysis of the measure $\nu$. Analytical and experimental results for the measure $\nu$ may be found in [6]. 


\section{WORST-CASE BOUNDS ON FABRICATION ERRORS}

The expressions for the second differential of $E_{s q}$ may be used to study the effect of a small change $\Delta M$ in the scanning filters. The absolute value of the change in a scalar function due to a small change of $\Delta \mathbf{M}$ in optimal scanning filters $M$, may be expressed as

$$
|\delta g| \approx\left|\frac{1}{2} d^{2} g(\operatorname{vec} \mathbf{M} ; \operatorname{vec} \Delta \mathbf{M})\right| \approx \frac{1}{2}(\operatorname{vec} \Delta \mathbf{M})^{T} \mathcal{H} \operatorname{vec} \Delta \mathbf{M}
$$

for the relevant matrix $\mathcal{H}$ derived in [6]. The matrix $\mathcal{H}$ is the Hessian of a function near a local minimum (of $E_{s q}$ ) and may hence be assumed positive semidefinite. Given the matrix $\mathcal{H}$, it is possible to calculate the maximum allowable fabrication errors for the scanning filters, given maximum allowable change in $g$. Three methods of perturbing the optimal filter designs will be investigated. The first one considers perturbing the filter design at a single wavelength of a single filter. The second considers perturbing the filter designs by the same amount at each wavelength for all filters. The third deals with perturbing the filter designs by a total error vector of fixed Euclidean norm.

Suppose that an error of $\pm \omega_{k}$ is made at the lth wavelength of the $m$ th filter, where $k=(m-1) N+l$ is the position of the error in the stacked vector vec $d \mathbf{M}$. Suppose further that all other fabrication errors are zero. Then

$$
|\delta g| \approx \frac{1}{2} \mathcal{H}_{k, k} \omega_{k}^{2} .
$$

As the matrix $\mathcal{H}$ is defined so as to be positive semidefinite, $\mathcal{H}_{k k}$ is nonnegative. The change in the value of $g$ is bounded by

$$
|\delta g| \leq \epsilon
$$

if $\omega_{k}$ satisfies

$$
\begin{gathered}
\left|\omega_{k}\right| \leq \sqrt{\frac{2 \epsilon}{\mathcal{H}_{k k}}} . \\
\stackrel{\text { def }}{=} \omega_{1}(k)
\end{gathered}
$$

Thus, the inequality (10) provides a bound for the allowable fabrication error as a function of wavelength. The bound of inequality (10) will be referred to as the single-wavelength bound. Note that isolated fabrication errors are not likely to occur. Hence, the single-wavelength bound does not provide a bound that is likely to represent a physical situation. Since the second differential is not a linear expression in the fabrication errors at individual wavelengths, the single-wavelength bound does not provide changes that can be added when fabrication errors occur at more than one wavelength. The advantage of the single-wavelength bound is that it provides a qualitative estimate of which wavelengths are most/least sensitive to fabrication errors.

Now suppose an error of $\pm \omega$ occurs at each wavelength of every filter. The bound for this case is given by

$$
|\delta g| \leq \frac{1}{2} \omega^{2} \sum_{i=1}^{r N} \sum_{j=1}^{r N}\left|\mathcal{H}_{i j}\right| .
$$

Hence, using the maximum variation for each element in the perturbation vector, $\Delta \mathbf{M}$, the change in the scalar error measure can be bounded by $\rho$, as follows:

$$
|\delta g| \leq \rho
$$

if

$$
\begin{aligned}
& \omega=\max _{i}\left(\left|\operatorname{vec} \Delta \mathbf{M}_{i}\right|\right) \\
& \leq \sqrt{\frac{2 \rho}{\sum_{i=1}^{r N} \sum_{j=1}^{r N}\left|\mathcal{H}_{i j}\right|}} \\
& \stackrel{\text { def }}{=} \omega_{2} \text {. }
\end{aligned}
$$

It is easy to see that the bound is achieved if the fabrication error at the $l$ th wavelength in the $m$ th filter has the sign of $\mathcal{H}_{i j}$ for all $j$ and $i=(m-1) N+l$. This is possible only if $\mathcal{H}_{i j}$ has the same sign for all $j$. As $\mathcal{H}$ is assumed positive semidefinite, nonzero diagonal values of $\mathcal{H}$ will be positive, which implies that the bound is tight if and only if all values of $\mathcal{H}$ are nonnegative. This is not always true. Inequality (11) relates the infinity norm of the error vector vec $\Delta \mathrm{M}$ to the maximum acceptable change in $E_{s q}$. This bound on the maximum change at all wavelengths of all filters will be referred to as the all-wavelength bound. This bound presents a more accurate bound, since errors at all wavelengths are considered. On the other hand, it does not provide any information about which wavelengths are more or less sensitive to fabrication errors.

In order to relate the change in $g$ to the 2-norm or Euclidean norm of the error vector, observe the following [1]:

$$
\frac{1}{2}(\operatorname{vec} \Delta \mathbf{M})^{T} \mathcal{H} \operatorname{vec} d \boldsymbol{\Delta} \mathbf{M} \leq \frac{1}{2}\|\operatorname{vec} \Delta \mathbf{M}\|^{2} \lambda_{\max }
$$

where $\lambda_{\max }$ is the largest eigenvalue of $\mathcal{H}$, and $\|$.$\| denotes$ the 2-norm or the Euclidean norm. This implies that

$$
\delta g \leq \gamma
$$

if the fabrication error $\Delta \mathbf{M}$ is such that

$$
\begin{aligned}
\|\operatorname{vec} \Delta \mathbf{M}\| & \leq \sqrt{\frac{2 \gamma}{\max _{i} \lambda_{i}}} \\
& \stackrel{\text { def }}{=} \omega_{3}
\end{aligned}
$$

where $\omega_{3}$ is defined as the Euclidean-norm bound. The bound $\gamma$ is achieved when vec $\Delta \mathbf{M}$ is an eigenvector of $\mathcal{H}$ corresponding to the largest eigenvalue.

It is possible to relate the single-wavelength and the allwavelength bounds as follows. Suppose $\delta g$ is the allowed change in $E_{s q}$ or in the data-independent measure $\nu$. Then, from (10) and (11) (using $\rho=\epsilon=\delta g$ )

$$
\omega_{1}(k)=\sqrt{\frac{\sum_{i=1}^{r N} \sum_{j=1}^{r N}\left|\mathcal{H}_{i j}\right|}{\mathcal{H}_{k k}}} \omega_{2}
$$

which implies that

$$
\omega_{1}(k) \geq \omega_{2}
$$


as expected. The allowable error at a single wavelength (represented by the single-wavelength bound $\omega_{1}$ ) should be larger than the allowable error at all wavelengths (represented by the all-wavelength bound $\omega_{2}$ ) for a fixed change in $E_{s q}$.

To relate the single-wavelength and all-wavelength bounds with the Euclidean-norm bound, consider the following. Suppose $\delta g$ is the allowed change in $E_{s q}$. Then, from (10) and (12) (where $\epsilon=\gamma=\delta g$ )

$$
\omega_{1}(k)=\sqrt{\frac{\lambda_{\max }}{\mathcal{H}_{k k}}} \omega_{3} .
$$

It is not possible to assign an ordering to these bounds without knowing the values of $\lambda_{\max }$ and $\mathcal{H}_{k \cdot k}$, unless the matrix $\mathcal{H}$ is diagonal. If $\mathcal{H}$ is diagonal, the values of $\mathcal{H}_{k k}$ are the eigenvalues of $\mathcal{H}$, and $\omega_{1}(k) \geq \omega_{3}$.

From (11) and (12) (where $\rho=\gamma=\delta g$ ),

$$
\omega_{2}=\sqrt{\frac{\lambda_{\max }}{\sum_{i=1}^{r N} \sum_{j=1}^{r N}\left|\mathcal{H}_{i j}\right|}} \omega_{3} .
$$

As $\mathcal{H}_{k k}$ is nonnegative, and the trace of a matrix is the sum of its eigenvalues, $\omega_{2} \leq \omega_{3}$. Thus, the all-wavelength bound is smaller than both the single-wavelength bound and the Euclidean-norm bound.

\section{EXPERIMENTAL RESULTS}

The filters in [7] were designed for scanner characteristics 1 and 2, $\mathbf{H}$ of (3) shown in Figs. 1 and 3 of [7], respectively. The second-order approximation to $E_{s q}$ is tested on the trimmed single-Gaussian filters for illuminant 1 and the trimmed double-Gaussian filters for illuminant 2 reported in [7]. Errors were estimated and computed for a set of filters that were obtained from Barr Associates, a filter manufacturer, as estimates of closest manufacturable interference filters in response to filter designs provided by the authors and reported in [6] and [7]. Errors were also estimated and computed for filters with randomly generated errors.

The single-wavelength bounds [inequality (10)] are tested for a change in $E_{s q}$ of 0.005 . The all-wavelength bound [inequality (11)] is calculated for a change in value of 1 in $E_{s q}$. The bound is tested on simulated filter sets. The filter sets are generated by the addition of a random sequence lying within the bound.

All errors were computed for simulated scanning of a 64data-point subset of the set of Munsell color chips [8] followed by data correction [5]. The white point used for the data set was the white sample of the Munsell set. As the CIE matching functions are the defining standards for the purpose of color scanning, it is of interest to compare the sensitivity of the designed filters to that of the CIE matching functions. The comparison indicates that the designed filters are no more sensitive than the CIE functions themselves.
TABLE I

Predicted and Actual Values of $E_{s q}$ : Fabrication Errors

\begin{tabular}{||c|c|c|c|c||} 
Set & $\begin{array}{c}\text { Original } \\
\text { Error }\end{array}$ & $\begin{array}{c}\text { Predicted } \\
\text { Error } \\
\text { Equation (9) }\end{array}$ & $\begin{array}{c}\text { Actual } \\
\text { Error }\end{array}$ & $\|v e c \Delta \mathrm{M}\|^{2}$ \\
\hline 1 & 0.1001 & 1.0486 & 1.2366 & 0.0440 \\
\hline 2 & 0.0322 & 0.4063 & 0.4088 & 0.0324 \\
\hline
\end{tabular}

TABLE II

Predicted and Actual Values of $E_{s q}$ : Simulated ErRors

\begin{tabular}{|c|c|c|c|c||}
\hline Set & $\begin{array}{c}\text { Original } \\
\text { Error }\end{array}$ & $\begin{array}{c}\text { Predicted } \\
\text { Error } \\
\text { Equation (9) }\end{array}$ & $\begin{array}{c}\text { Actual } \\
\text { Error }\end{array}$ & $\|$ vec $\Delta \mathbf{M} \|^{2}$ \\
\hline 1 & 0.1001 & 0.2072 & 0.2717 & 0.0146 \\
\hline 1 & 0.1001 & 0.3012 & 0.3398 & 0.0208 \\
\hline 1 & 0.1001 & 0.1631 & 0.1936 & 0.0097 \\
\hline 2 & 0.0322 & 0.3970 & 0.4201 & 0.0818 \\
\hline 2 & 0.0322 & 0.2130 & 0.2249 & 0.0106 \\
\hline 2 & 0.0322 & 0.1912 & 0.2024 & 0.0170 \\
\hline
\end{tabular}

\section{A. The Second-Order Approximation}

The trimmed single-Gaussian filters for illuminant 1 are denoted filter set 1 . The trimmed sum-of-Gaussian filters for illuminant 2 are denoted filter set 2 . The CIE matching functions under uniform illumination, normalized to a maximum value of unity to facilitate comparison with realizable filter transmissivities, are denoted filter set 3 .

Consider the value of $\Delta \mathbf{M}$ defined by the difference between the filter sets 1 and 2 and their respective realizable filters that Barr Associates can manufacture, which are presented in [7]. Using this vector $\Delta \mathbf{M},(9)$ is used to predict the change in $E_{s q}$. This prediction is compared to the actual computed change in Table I. The bound of $E_{s q}$ calculated from the Euclidean norm of vec $\Delta \mathrm{M}$ is 7.0 for filter set 1 and 28.28 for filter set 2 . This bound is much larger than the actual error and does not prove to be valuable in assessing changes in the $\Delta E_{a b}$ error. The original error in the table refers to the $E_{s q}$ of the unperturbed filters ( $\sqrt{E_{s q}}$ is RMS of Table V of [7]).

As the generation of realizable sets involves the use of proprietary computer modeling programs, it is not possible to generate a large number of realizable filters to test the secondorder approximation. In order to further test the approximation, sets were generated by the addition of white Gaussian noise with a variance corresponding to the variation observed in the realizable filters. Table II lists typical predicted and actual errors for the trimmed filter sets 1 and 2, and randomly generated actual filter sets. The results tabulated in Tables I and II indicate that the second differential provides a fair estimate of the effect of fabrication error on $E_{s q}$. The estimate is slightly lower than the actual values. The reason for the discrepancy is that the higher-order derivatives contribute a nonnegligible amount of the change or that the norm of the error vector is not small enough.

\section{B. Single-Wavelength Bounds}

Single-wavelength bounds calculated from (10) for $\epsilon=$ 0.005 are plotted as a function of wavelength in Figs. 1-6 for 


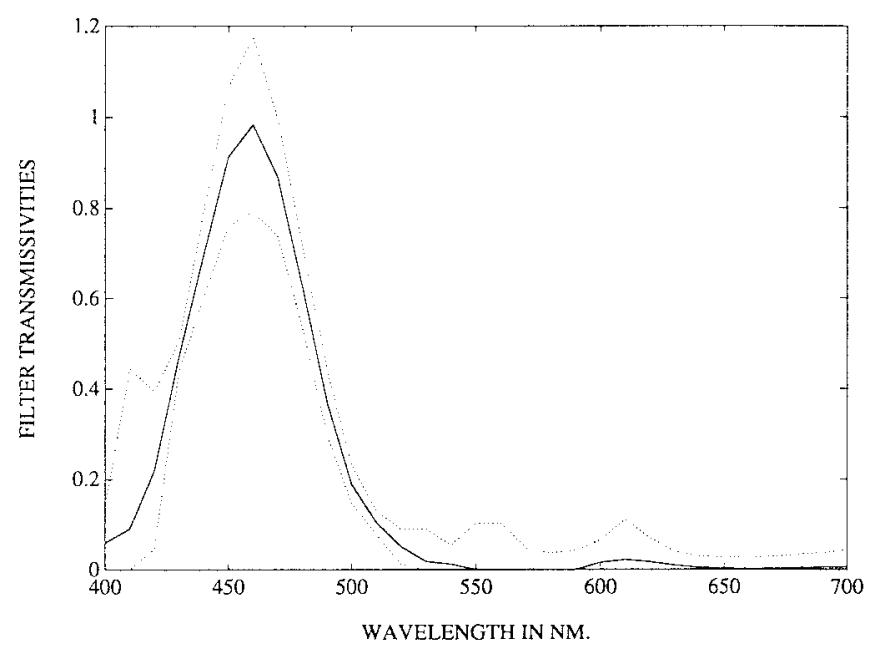

Fig. 1. Single-wavelength bounds for blue filter of trimmed single Gaussian model and illuminant $1, \epsilon=0.005 . \cdots$ upper and lower bounds, -- trimmed filter.

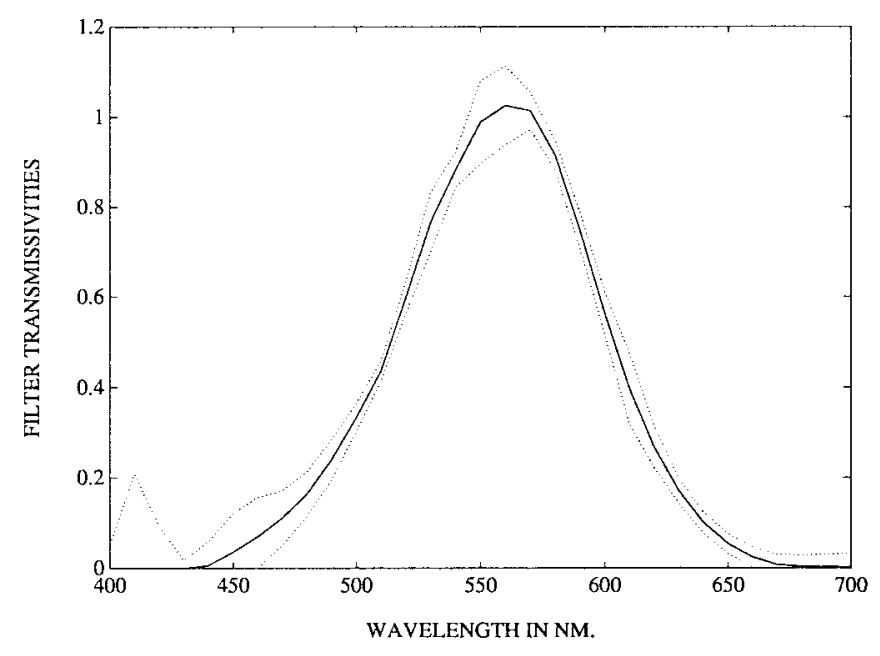

Fig. 2. Single-wavelength bounds for green filter of trimmed single Gaussian model and illuminant $1, \epsilon=0.005 . \cdots$ upper and lower bounds, _- trimmed filter.

filter sets 1 and 3. The dotted lines indicate the bounds, and the solid line indicates the designed filter in all plots. Table III lists the maximum and minimum value of the perturbations plotted for each of the designed filter sets. The filters and the wavelengths at which the respective errors may occur are also listed. This allows comparison of the sensitivities of the two sets of filters. The values of the maximum and minimum allowable perturbations are similar for the two sets of filters, which implies similar sensitivities.

Notice that the minimum allowable error at a single wavelength is higher for the single-Gaussian design than for the CIE matching functions. Further, the sum-of-Gaussian design allows a very large maximum value of fabrication error as compared to that allowed by the CIE matching functions. This indicates that the single-Gaussian and sum-of-Gaussian designs are not unduly sensitive.

To test the validity of the single-wavelength bounds, the trimmed filter sets were perturbed at exactly one wavelength

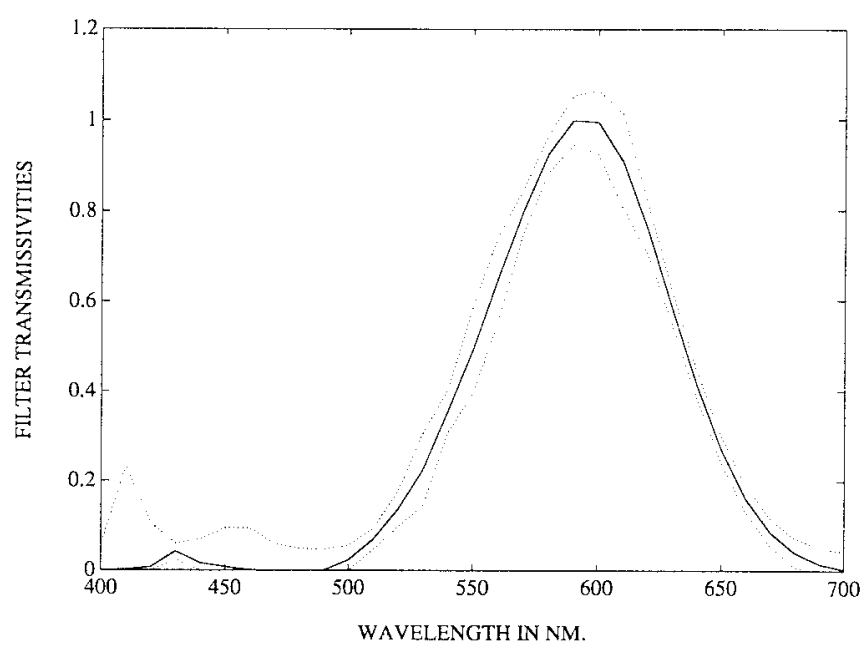

Fig. 3. Single-wavelength bounds for red filter of trimmed single Gaussian model and illuminant $1, \epsilon=0.005$. . u upper and lower bounds, _- trimmed filter.

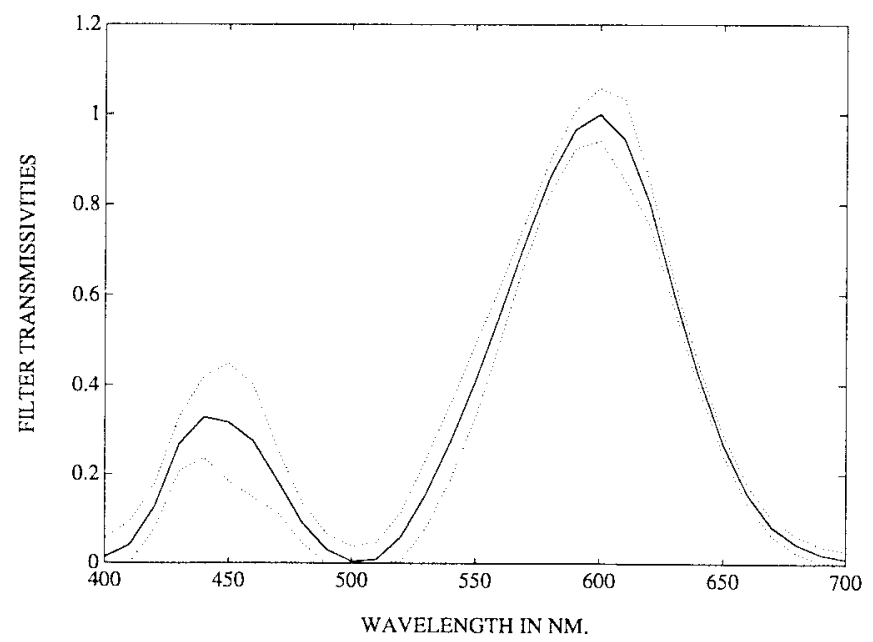

Fig. 4. Single-wavelength bounds for blue filter of the CIE functions under a uniform illuminant, $\epsilon=0.005 . \cdots$ upper and lower bounds, _- CIE function.

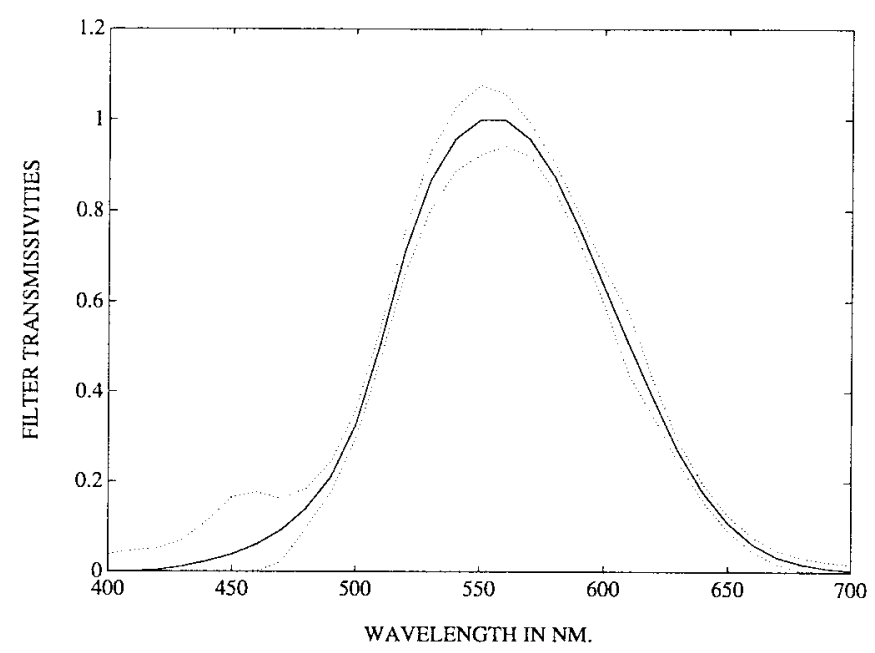

Fig. 5. Single-wavelength bounds for green filter of the CIE functions under a uniform illuminant, $\epsilon=0.005 . \cdots$ upper and lower bounds, _- CIE function. 


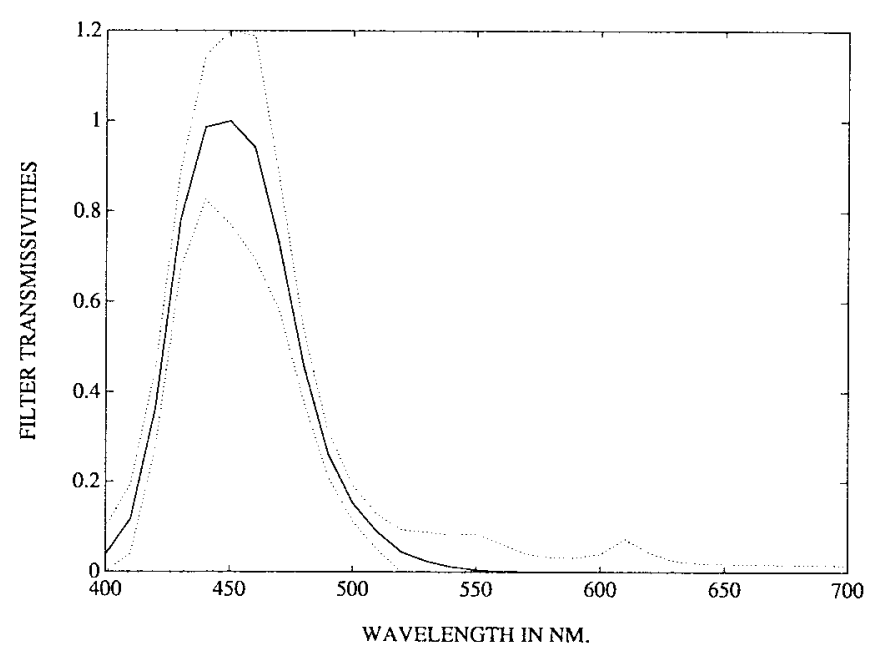

Fig. 6. Single-wavelength bounds for red filter of the CIE functions under a uniform illuminant, $\epsilon=0.005 . \cdots$ upper and lower bounds, ${ }_{--}$CIE function.

TABLE III

MaXimum AND Minimum Values of THE SiNGLE-WAVELENGTH BOUND FOR $\epsilon=0.005$

\begin{tabular}{|c|c|c|c|c||}
\hline Set & & Perturbation & Filter & Wavelength in $\mathrm{nm} . \mid$ \\
\hline \multirow{2}{*}{1} & Minimum & 0.0174 & Green & 430 \\
\cline { 2 - 5 } & Maximum & 0.3538 & Blue & 410 \\
\hline \multirow{2}{*}{2} & Minimum & 0.0045 & Red & 500 \\
\cline { 2 - 5 } & Maximum & 7.4102 & Green & 680 \\
\hline \multirow{2}{*}{3} & Minimum & 0.0123 & Blue & 700 \\
\cline { 2 - 5 } & Maximum & 0.2031 & Blue & 460 \\
\hline
\end{tabular}

by an amount equal to the single-wavelength bound at that wavelength, corresponding to a change of 0.005 . The value of $E_{s q}$ for the perturbed filter set was calculated. This was done for each wavelength and each filter set. The largest value of $E_{s q}$ on perturbation for filter set 1 was 0.1255 , which is slightly larger than the bound for this set, which is 0.1051 . This error occurred at wavelength $400 \mathrm{~nm}$ in the blue filter. The largest value for filter set 2 was 0.0502 at $700 \mathrm{~nm}$, for the red filter, for a bound of 0.0372 . For filter set 3 , the largest value was 0.0054 for a bound of 0.005 , at $440 \mathrm{~nm}$ for the red filter.

There were many wavelengths in each filter where the value of $E_{s q}$ was larger than the bound. In some of these cases, the norm of the vector vec $\Delta \mathbf{M}$ was large enough to indicate that the Taylor series approximation of the second order was insufficient. In cases where the norm is not particularly high, it is suspected that the values of the higher derivatives are large enough at these points to make a second-order Taylor approximation invalid. In any case, an approximation that is within $25 \%$ of the bound is still quite useful.

\section{All-Wavelength Bound}

The all-wavelength bounds indicated by (11) are calculated for a maximum allowable change of 1 in $E_{s q}$. A representative plot is presented in Fig. 7. Table IV lists the error measures for the original designs and the corresponding designs perturbed within the bounds. The mean square $\Delta E_{a b}$ error, $E_{s q}$, is

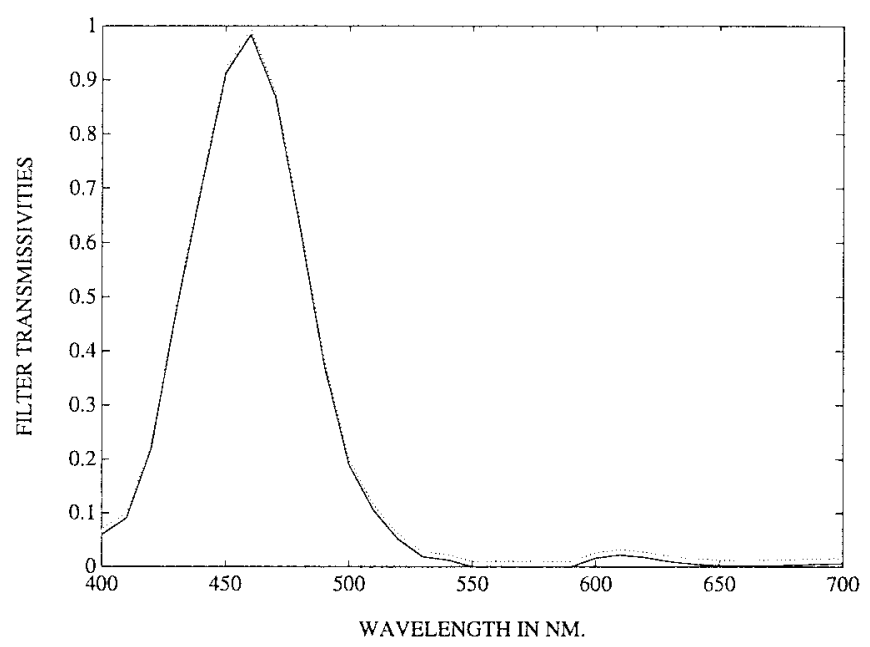

Fig. 7. All-wavelength bound for trimmed blue filter of single Gaussian model and illuminant $1, \rho=1 . \cdots$ upper and lower bounds, _- trimmed filter

TABLE IV

Comparison Between Errors of Original Design and Perturbed Design for a Predicted Change of 1 IN $E_{s q}$

\begin{tabular}{|c|c|c|c|c|c|c|c|c|c|}
\hline Set & & v & $F$ & $E_{\text {traus: }}$ & RVIS & $\mathrm{N} 1 \mathrm{~S}$ & P.IS & $\omega_{2}$ & ||vec $\Delta \mathrm{MI}:^{2}$ \\
\hline \multirow[t]{2}{*}{1} & Original & 0.9508 & 0.27 & 0.67 & 0.32 & 0.10 & 0.10 & 0 & 0 \\
\hline & Perturbed & 0.9484 & 0.71 & 2.63 & 0.91 & 0.83 & 1.10 & 0.010 & 0.0093 \\
\hline \multirow[t]{2}{*}{2} & Original & 0.0918 & 0.12 & 0.73 & 0.18 & 0.03 & 0.03 & 0 & 0 \\
\hline & Perturbed & 0.9589 & 0.66 & 3.43 & 0.93 & 0.86 & 1.03 & 0.007 & 0.0046 \\
\hline \multirow[t]{2}{*}{3} & Original & 1.0 & 0 & 0 & 0 & 0 & 0 & 0 & 0 \\
\hline & Perturbed & 0.9993 & 0.57 & 2.99 & 0.81 & 0.65 & 1.0 & 0.008 & 0.0059 \\
\hline
\end{tabular}

denoted MS. The predicted value of $E_{s q}$ (the bound corresponding to the perturbation) is denoted PMS. The calculated all-wavelength bound is denoted $\omega_{2}$. Note that the bounds plotted represent the same fabrication error at each wavelength, though at some points it appears as though the bounds are unequal because of different values of slopes in the graphs. Recall that the all-wavelength bound does not signify an error bound that is necessarily tight.

The realizable filters for filter set 1 indicate a maximum manufacturing error of value 0.06 with respect to the trimmed filters. The filters that can be used to match filter set 2 also indicate a maximum manufacturing error of 0.06 . This value is much higher than the all-wavelength bound for a change of value 1 in $E_{s q}$. It is noted in [7], however, that the changes in the perceptual error and in the measure due to the fabrication error are negligible. The realizable filters perform well. This is not surprising, since the bounds represent worst-case situations.

All the computations of the bounds indicate that the designed filters are highly sensitive because the allowable filter errors computed are far smaller than real fabrication errors. The experiments also indicate that the designed filters are not any more sensitive than the CIE matching functions. Given that the cone sensitivities vary considerably among individuals [3], [4], small variations in scanning filters may be acceptable if visual output is the final criterion, because the human color sensors vary considerably themselves. On the other hand, if quantitative color as measured by an electro- 
optical instrument is the final criterion, the small variations may make a difference.

\section{CONCLUSIONS}

The sensitivity of color scanning filters to fabrication errors was studied. The degradation in performance of the filter sets was approximated by the second-order term of the Taylor series expansion of the optimization criteria used to design the filters. The approximation provided estimates of error sensitivity as a function of wavelength. The approximation was used to obtain bounds on allowable filter fabrication errors given maximum acceptable changes in the optimization criterion. Experiments were performed to check the accuracy of the analysis with $E_{s q}$ as the optimization criterion. The experiments demonstrated that the bounds thus obtained were reasonable indicators of the performance degradation. The experiments also indicated that the designed filters were highly sensitive to fabrication errors. The fact that the CIE matching functions displayed similar sensitivities to fabrication error implied that the sensitivity could be characteristic of the scanning process.

\section{ACKNOWLEDGMENT}

The authors would like to thank Barr Associates for their help with this research.

\section{REFERENCES}

[1] G. H. Golub and C. F. Van Loan, Matrix Computations. Baltimore, MD: Johns Hopkins Univ. Press, 1989.

[2] J. R. Magnus and H. Neudecker, Matrix Differential Calculus with Applications in Statistics and Econometrics. New York: Wiley, 1988.

[3] A. D. North and M. D. Fairchild, "Measuring color matching functions, part II: New data for assessing observer metamerism," Color Res. Appl., vol. 18, pp. 163-170, 1993.

[4] V. C. Smith and J. Pokorny, "Chromatic-discrimination axes, CRT phosphor spectra, and individual variation in color vision," J. Opt. Soc. Amer. A, vol. 12, pp. 27-35, 1995.

[5] P. L. Vora and H. J. Trussell, "Measure of goodness of a set of color scanning filters," J. Opt. Soc. Amer. A, vol. 10, no. 7, pp. 1499-1508, July 1993.

[6] P. L. Vora, "Optimization criteria and numerical analysis in the design of color scanning filters," Ph.D. dissertation, North Carolina State Univ., Raleigh, 1993.

[7] P. L. Vora and H. J. Trussell, "Mathematical methods for the design of color scanning filters," IEEE Trans. Image Processing, this issue, pp. $312-320$.

[8] M. J. Vrhel, R. Gershon, and L. Iwan, "The measurement and analysis of object reflectance spectra," Color Res. Applic., vol. 19, pp. 4-9, Feb. 1994.

[9] G. Wyszecki and W. S. Stiles, Color Science: Concepts and Methods, Quantitative Data and Formulae. New York: Wiley, 1982.

Poorvi L. Vora, for photograph and biography, see this issue, p. 320.

H. Joel Trussell (S'75-M'76-SM'91-F'94), for photograph and biography, see this issue, p. 320. 